\title{
New Methods with Capped Options for Pricing American Options
}

\author{
Dongya Deng ${ }^{1}$ and Cuiye Peng ${ }^{2}$ \\ ${ }^{1}$ School of Finance, Southwestern University of Finance and Economics, Chengdu 611130, China \\ ${ }^{2}$ Library, Southwestern University of Finance and Economics, Chengdu 611130, China \\ Correspondence should be addressed to Dongya Deng; 112020204015@2012.swufe.edu.cn
}

Received 14 January 2014; Revised 26 February 2014; Accepted 28 March 2014; Published 27 April 2014

Academic Editor: Jin Liang

Copyright (c) 2014 D. Deng and C. Peng. This is an open access article distributed under the Creative Commons Attribution License, which permits unrestricted use, distribution, and reproduction in any medium, provided the original work is properly cited.

\begin{abstract}
We propose two new methods: improved binomial methods and improved least square MonteCarlo methods (LSM), for pricing American options. These two methods are developed using the nice capped options which have closed-form formulas. Numerical examples are provided to verify that these two new methods are pretty efficient.
\end{abstract}

\section{Introduction}

American style options are very important in hedging instruments. The binomial tree and least square Monte Carlo method (LSM) are two popularly used methods for pricing American options. Binomial tree was first introduced by Cox et al. [1] and developed by many researchers (see, e.g., a book by Kwok [2]). Least square Monte Carlo method was originally invented by Longstaff and Schwartz [3] and became well-known and popular approaches after then.

In this paper it is found that the binomial tree methods and least square Monte Carlo methods can be greatly improved by nice option-capped options with closed-form solutions. Capped option is a conventional option with a predefined profit cap written into the contract. A capped option is automatically exercised when the underlying security closes at a price making the option's mark to market match the specified amount. Boyle and Turnbull [4] provided the valuation formulas for European capped options and Broadie and Detemple [5] gave the closed-form formula for American capped option under the condition of low dividend.

In this paper we use the American capped option with the closed-form formula given by Broadie and Detemple [5] to improve the binomial tree approach for pricing American call options. Also we develop new improvement to the least square Monte Carlo method using the lower bound on the early exercise boundary which was provided by Broadie and Detemple [6]. Numerical examples are given to confirm our findings.

The paper is organized as follows. In Section 2 we introduce capped options. In Sections 3 and 4 we introduce how to use capped options to improve the traditional binomial tree method and LSM. In Section 5 we give the result of numerical implementations. Conclusions and remarks on the future work are given in the last section.

\section{Capped Options}

Let $C_{t}^{L}$ represent the value of an American capped call option at time $t$. The American capped call option has a strike price $K$, a cap $L$, a risk free rate $r$, a low dividend $\delta$, and a maturity $T$. Throughout the paper, we assume that $L \geq K>0$. Exercise may take place, at the discretion of the owner of the security, at any date during the life of the option $[0, T]$. The capped call option with payoff $\max \left(\min \left(L, S_{t}\right)-K, 0\right)$ and condition $\delta \leq r K / L$ is given by (see Broadie and Detemple [6])

$$
\begin{aligned}
C_{t}^{L} & \left(S_{t}, L\right) \\
\quad & =(L-K)\left[\lambda_{t}^{2 \phi / \sigma^{2}} N\left(d_{0}\right)+\lambda_{t}^{2 \alpha / \sigma^{2}} N\left(d_{0}+\frac{2 f \sqrt{T-t}}{\sigma}\right)\right]
\end{aligned}
$$




$$
\begin{gathered}
+S_{t} e^{-\delta(T-t)}\left[N\left(d_{1}^{-}(L)-\sigma \sqrt{T-t}\right)\right. \\
\left.-N\left(d_{1}^{-}(K)-\sigma \sqrt{T-t}\right)\right] \\
-\lambda_{t}^{-\left(2(r-\delta) / \sigma^{2}\right)} L e^{-\delta(T-t)}\left[N\left(d_{1}^{+}(L)-\sigma \sqrt{T-t}\right)\right. \\
\left.-N\left(d_{1}^{+}\left(S_{t}\right)-\sigma \sqrt{T-t}\right)\right] \\
-K e^{-r(T-t)}\left[N\left(d_{1}^{-}(L)\right)-N\left(d_{1}^{-}(K)\right)-\lambda_{t}^{1-\left(2(r-\delta) / \sigma^{2}\right.}\right) \\
\left.\times\left[N\left(d_{1}^{+}(L)\right)-N\left(d_{1}^{+}(K)\right)\right]\right]
\end{gathered}
$$

where

$$
d_{0}=\frac{1}{\sigma \sqrt{T-t}}\left[\log \left(\lambda_{t}\right)-f(T-t)\right]
$$

$$
\begin{aligned}
& d_{1}^{ \pm}(x) \\
& =\frac{1}{\sigma \sqrt{T-t}}\left[ \pm \log \left(\lambda_{t}\right)-\log (L)+\log (x)+b(T-t)\right] \\
& b=\delta-r+\frac{1}{2} \sigma^{2} \\
& f=\sqrt{b^{2}+2 r \sigma^{2}} \\
& \phi=\frac{1}{2}(b-f), \\
& \alpha=\frac{1}{2}(b+f), \quad \lambda_{t}=\frac{S_{t}}{L} .
\end{aligned}
$$

$N(\cdot)$ denotes the cumulative standard normal distribution. The procedure relies heavily on the derivative of the capped call option value with respect to the constant cap $L$, evaluated as $S_{t}$ approaches $L$ from below:

$$
D(L, t) \equiv \lim _{S_{t} \downarrow L} \frac{\partial C_{t}^{L}\left(S_{t}, L\right)}{\partial L},
$$

$$
\begin{aligned}
& \frac{\partial C_{t}^{L}\left(S_{t}, L\right)}{\partial L} \\
& =\left[1-\left(\frac{L-K}{L}\right)\left(\frac{2 \phi}{\sigma^{2}}\right)\right] \lambda_{t}^{2 \phi / \sigma^{2}} N\left(d_{0}\right) \\
& \quad+\left[1-\left(\frac{L-K}{L}\right)\left(\frac{2 \alpha}{\sigma^{2}}\right)\right] \lambda_{t}^{2 \alpha / \sigma^{2}} N\left(d_{0}+\frac{2 f \sqrt{\tau}}{\sigma}\right) \\
& \quad+\lambda_{t}^{-\left(2(r-\delta) / \sigma^{2}\right)} e^{-\delta \tau} \frac{2\left(b-\sigma^{2}\right)}{\sigma^{2}} \\
& \quad \times\left[N\left(d_{1}^{+}(L)-\sigma \sqrt{\tau}\right)-N\left(d_{1}^{+}(K)-\sigma \sqrt{\tau}\right)\right] \\
& \quad-\lambda_{t}^{-\left(2 b / \sigma^{2}\right)} e^{-r \tau} \frac{2 b K}{\sigma^{2} L}\left[N\left(d_{1}^{+}(L)\right)-N\left(d_{1}^{+}(K)\right)\right] .
\end{aligned}
$$

So

$$
\begin{aligned}
D(L, t)= & {\left[1-\left(\frac{L-K}{L}\right)\left(\frac{2 \phi}{\sigma^{2}}\right)\right] N\left(-\frac{f \sqrt{\tau}}{\sigma}\right) } \\
& +\left[1-\left(\frac{L-K}{L}\right)\left(\frac{2 \alpha}{\sigma^{2}}\right)\right] N\left(\frac{f \sqrt{\tau}}{\sigma}\right) \\
& +e^{-\delta \tau} \frac{2\left(b-\sigma^{2}\right)}{\sigma^{2}} \\
& \times\left[N\left(d_{1}^{+}(L)-\sigma \sqrt{\tau}\right)-N\left(d_{1}^{+}(K)-\sigma \sqrt{\tau}\right)\right] \\
& -e^{-r \tau} \frac{2 b K}{\sigma^{2} L}\left[N\left(d_{1}^{+}(L)\right)-N\left(d_{1}^{+}(K)\right)\right], \\
\frac{\partial D(L, t)}{\partial L}= & -\frac{K}{L^{2}}\left[\frac{2 \phi}{\sigma^{2}}+\frac{2 f}{\sigma^{2}} N\left(\frac{f \sqrt{\tau}}{\sigma}\right)\right] \\
& -2 e^{-\delta \tau} \frac{n\left(d_{1}^{+}(L)-\sigma \sqrt{\tau}\right)}{L \sigma \sqrt{\tau}} \\
& -e^{-r \tau} \frac{2 b K}{\sigma^{2} L^{2}}\left[N\left(d_{1}^{+}(L)\right)-N\left(d_{1}^{+}(K)\right)\right] .
\end{aligned}
$$

Denote by $L^{*}$ the solution to the equation

$$
D\left(L^{*}, t\right)=0 .
$$

Equation (5) represents a zero-finding problem that can be solved easily, for example, using Newton's method with (4).

\section{New Binomial Algorithm for American Options}

In this section, how to use American capped option in improving binomial tree method is introduced. Binomial tree is a classical method. Cox et al. [1] introduced binomial tree method. Throughout the following paper, suppose that the constant interest rate $r>0$ and the constant volatility $\sigma>0$ are given, and continuous capital markets are modeled by a stock price process $S_{t}$ following geometric Brownian motion

$$
d S_{t}=S_{t}(r-\delta) d t+S_{t} \sigma d W_{t}
$$

where $W_{t}$ is a standard Wiener process on some probability space $(X, F, Q)$. Suppose $C^{A}\left(S_{t}\right)$ is the value of American call option for the continuous-time Black-Scholes model, where $S_{t}$ is the asset's price at time $t$, between time $[0, T]$. The American call option can be written as

$$
C^{A}\left(S_{t}\right)=\sup _{\tau \in[0, T]} E_{t}\left[e^{-r \tau} C\left(S_{\tau}\right)\right],
$$

where $\tau$ is the stopping time, and $C\left(S_{\tau}\right)=\max \left(S_{\tau}-K, 0\right)$. $E_{t}[\cdot]$ is the conditional expectation at $t$ under risk neutral probability measure.

Binomial models are the description of discrete asset price dynamics. They specify a number $n$ of trading dates. Trading occurs only at the equidistant spots of time $t_{i} \in[0, T]$, $i=0, \ldots, n$, and $0=t_{0}<t_{1}<\cdots<t_{n}=T$. In order to achieve a complete market model, the one-period 
return $\bar{R}_{i}(i=0, \ldots, n)$ is modeled by two point i.i.d binomial random variables

$$
\bar{R}_{i}= \begin{cases}u_{n} \text { with probability } & q_{n} \\ d_{n} \text { with complementary probability } & 1-q_{n}\end{cases}
$$

on a suitable probability space $(\bar{X}, \bar{F}, \bar{Q})$. Therefore the discrete asset price dynamics is $\bar{S}_{k}$, where the price $\bar{S}_{t_{k}}$ at time $t_{k}$ is described by

$$
\bar{S}_{t_{k}}=S_{0} \prod_{i=1}^{k} \bar{R}_{i}
$$

The specification of the one-period returns is a complete description of the discrete dynamics. The model of CRR uses

$$
u_{n}=e^{\sigma \sqrt{\Delta t_{n}}}, \quad d_{n}=e^{-\sigma \sqrt{\Delta t_{n}}},
$$

where $\Delta t_{n}=T / n$ is the stepsize. To take into account the riskneutrality argument of Harrison and Pliska [7], the expected one-period return $\bar{E}\left[\bar{R}_{i}\right]$ must be equal to the one-period return of the riskless bond $r_{n}=e^{r \Delta t_{n}}$. This amounts to setting $\bar{q}_{n}=\left(r_{n}-d_{n}\right) /\left(u_{n}-d_{n}\right)$. The risk-neutrality argument amounts to matching discrete and continuous first moments. In Tian [8], the parameter selection requires the second and third moments to be equal, too:

$$
\begin{aligned}
& u_{n}=\frac{r_{n} v_{n}}{2}\left(v_{n}+1+\sqrt{v_{n}^{2}+v_{n}-3}\right), \\
& d_{n}=\frac{r_{n} v_{n}}{2}\left(v_{n}+1-\sqrt{v_{n}^{2}+v_{n}-3}\right),
\end{aligned}
$$

where $v_{n}=e^{\sigma^{2} \Delta t_{n}}$

Denote the value of American call option calculated by the standard CRR binomial tree at time $t_{k}$ by $C^{B}\left(\bar{S}_{t_{k}}\right)$, the exercise value by $C^{e}\left(\bar{S}_{t_{k}}\right)$, and the hold-on value by $C^{h}\left(\bar{S}_{t_{k}}\right)$. It is known that $C^{B}\left(\bar{S}_{T}\right)=\max \left(\bar{S}_{T}-K, 0\right), C^{e}\left(\bar{S}_{t_{k}}\right)=\max \left(\bar{S}_{t_{k}}-\right.$ $K, 0)$, and $C^{h}\left(\bar{S}_{t_{k}}\right)=E_{t_{k}}\left(e^{-r \Delta t_{n}} C^{B}\left(\bar{S}_{t_{k+1}}\right)\right)$. Then standard CRR binomial value of American call option at $t_{k}$ is $C^{B}\left(\bar{S}_{t_{k}}\right)=$ $\max \left(C^{e}\left(\bar{S}_{t_{k}}\right), C^{h}\left(\bar{S}_{t_{k}}\right)\right)$.

The above procedure described the standard CRR binomial tree approach for calculation of American call option. Now we present the improved binomial tree approach. Denote $C^{E}(S)$ by the value of a European call option calculated by Black-Scholes formula. Broadie and Detemple [6] improved the binomial tree approach by replacing $C^{B}\left(\bar{S}_{t_{n-1}}\right)$ with $C^{E}\left(\bar{S}_{t_{n-1}}\right)$ at $t_{n}$, the time before maturity $T$. We find that this kind of improvement can be further refined. Since

$$
C^{E}\left(\bar{S}_{t_{n-1}}\right) \leq C_{t_{n-1}}^{L}\left(\bar{S}_{t_{n-1}}, L\right) \leq C^{A}\left(\bar{S}_{t_{n-1}}\right),
$$

where $C^{A}\left(\bar{S}_{t_{n-1}}\right)$ is the real value of American call option (see Broadie and Detemple [6]), which means that the value of capped option is much closer to the true American option than the European option, it is more accurate to replace $C^{B}\left(\bar{S}_{t_{n-1}}\right)$ by $C_{t_{n-1}}^{L}\left(\bar{S}_{t_{n-1}}, L\right)$. The cap $L$ can be chosen by

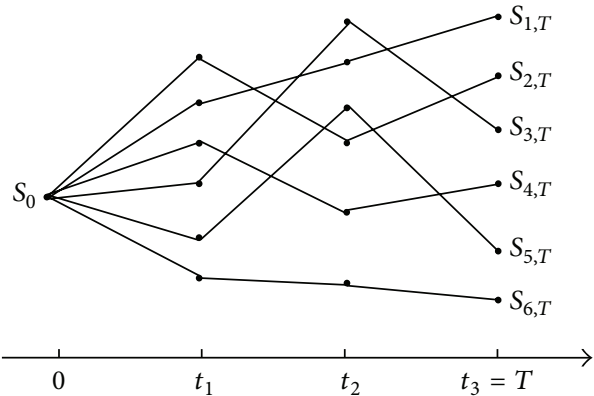

Figure 1: Simulation paths.

a practicer. In fact it is much more accurate to use the optimal value $L^{*}$ obtained by solving the nonlinear equation (5). Although it takes time to solve the nonlinear equation (5), the calculated result can be stored in computer and used for next time calculation. In this way the traditional binomial approach is greatly improved and accelerated.

\section{New LSM for American Options}

Least Monte Carlo method (LSM) was first introduced by Longstaff and Schwartz [3]. It is a simple yet powerful approach for approximating the value of American options by simulation. They only used in-the-money paths since it allows them to better estimate the conditional expectation function in the region where exercise is relevant and significantly improves the efficiency of the algorithm.

The key insight underlying LSM approach is that this conditional expectation can be estimated from the crosssectional information in the simulation by using least squares. Specifically, LSM regresses the realized payoffs from continuation on functions of the values of the state variables. The fitted value from this regression provides a direct estimate of the conditional expectation function. By estimating the conditional expectation function for each exercise date, LSM obtains a complete specification of the optimal exercise strategy along each path. With this specification, American options can then be valued accurately by simulation.

Suppose a stock price process $S_{t}$ following geometric Brownian motion as well. The initial price is $S_{0}$, and $S_{t}=$ $S_{t} e^{\left(r-(1 / 2) \sigma^{2}\right) t+\sigma W_{t}}$, where $W_{t} \sim N(0,1)$. By specifying a number $N$ of trading dates, $i=1, \ldots, N, j=1, \ldots, N$, and $j<i$, the discrete time is $0<t_{j}<\cdots<t_{i} \leq t_{N}=T$. Then, we can have

$$
S_{t_{i}}=S_{t_{j}} e^{\left(r-(1 / 2) \sigma^{2}\right)\left(t_{i}-t_{j}\right)+\sigma \sqrt{t_{i}-t_{j}} W_{t_{i}}} .
$$

The simulation paths are as shown in Figure 1.

There are six paths at Figure 1. The value of European call option is

$$
\begin{aligned}
C^{E}=\frac{1}{6} e^{-r T}( & \left(S_{1, T}-K\right)^{+}+\left(S_{2, T}-K\right)^{+}+\left(S_{3, T}-K\right)^{+} \\
& \left.+\left(S_{4, T}-K\right)^{+}+\left(S_{5, T}-K\right)^{+}+\left(S_{6, T}-K\right)^{+}\right) .
\end{aligned}
$$




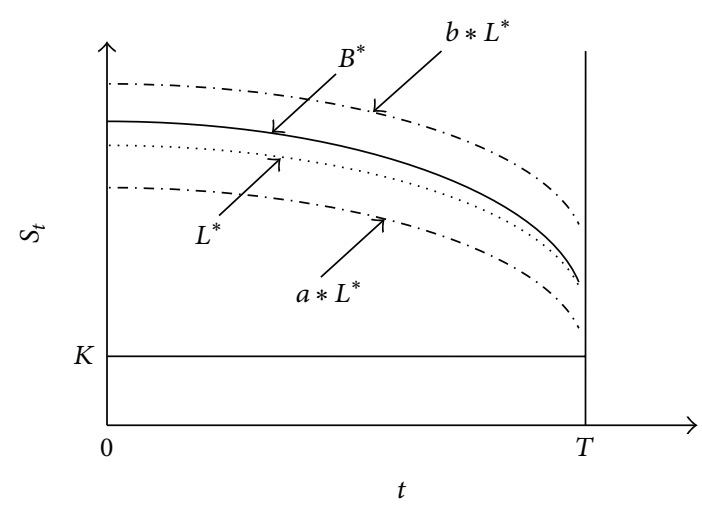

FIGURE 2: Lower bound on the early exercise boundary.

As American option can be excised at any time before maturity time $T$, the values of American option at any time $t$ also need to be compared as the procedure described in Section 3. Suppose there are total number of $P$ paths, among which the number of effective paths is $P^{\prime}$, where the effective paths are defined by the hold-on value that is above zero to regression (see Longstaff and Schwartz [3]).

LSM uses regression to obtain the regressed hold-on value. The estimated conditional expectation function is

$$
\begin{array}{r}
E\left(C^{h}\left(S_{k^{\prime}, t_{i}}\right) \mid S_{k^{\prime}, t_{i}}\right)=a+b * S_{k^{\prime}, t_{i}}+c * S_{k^{\prime}, t_{i}}^{2}, \\
k^{\prime}=1, \ldots, P^{\prime},
\end{array}
$$

where $P^{\prime}$ is the number of in-the-money paths, and $S_{k^{\prime}, t_{i}}$ is the price of underlying asset on the in-the-money path. The regressed hold-on value $\widehat{C}^{h}\left(S_{k, t_{i}}\right)$ is compared with excised value.

Therefore there exists stopping time at each path and a stopping matrix $P \times N$. With this specification of the stopping matrix, it is straightforward to determine the cash flows realized by following the stopping time. Having identified the cash flows generated by the American call at each date along each path, the value of American call option can now be valued by discounting each cash flow back to time zero and averaging over all. One possible choice of basis functions is the set of (weighted) Laguerre polynomials. Other types of basis functions include the Hermite, Legendre, Chebyshev, and Jacobi polynomials.

But there are some useless paths which raise the cost of computation in the Monte Carlo regression. In this paper we develop a new algorithm. More precisely we use a more accurate early exercise policy to recognize the paths that are not used in the Monte Carlo regression. Broadie and Detemple [6] used capped option as a tool to obtain the lower bond $L^{*}$ of American call option as shown in Figure 2, where the solid line $B^{*}$ is the optimal exercise boundary for an American call option. The dotted line $L^{*}$ is the lower bound (see (5)). Assume that there are $P^{\prime}$ in-the-money paths in Monte Carlo simulation for pricing a call option. Given a lower bound, we can filter out the invalid path in Monte Carlo simulation. We take interval $\left[a * L^{*} b * L^{*}\right]$ to reduce the number of paths, where $a$ and $b$ are two user-selected constants satisfying $b>1,0<a<1$, and use the paths on the interval to regression. Assume that there are $P^{*}$ in-themoney paths between the intervals, and obviously $P^{*}<P^{\prime}$; the new conditional expectation function is

$$
\begin{array}{r}
E\left(\widehat{C}^{h}\left(S_{k^{*}, t_{i}}\right) \mid S_{k^{*}, t_{i}}\right)=a^{*}+b^{*} * S_{k^{*}, t_{i}}+c^{*} * S_{k^{*}, t_{i}}^{2}, \\
k^{*}=1, \ldots, P^{*} .
\end{array}
$$

So, there is a new stopping rule and stopping matrix $P \times N$. In this way the traditional LSM method is greatly improved and accelerated.

\section{Numerical Analysis}

5.1. New Binomial Algorithm. In Examples 1, 2, and 4, the dividend rate is not lower than the interest rate. Broadie and Detemple [5] only proved formula (1) for the capped option with lower dividend rates. However the numerical results in these examples show that our algorithm is still correct using formula (1).

Example 1. Use capped option as a tool to improve CRR model with parameters: $S 0=100 ; \delta=0.07 ; r=0.03$; $\sigma=0.2 ; T=0.5 ; t=0 ; K=100$. The benchmark value for the American option calculated by CRR binomial tree with 50,000 steps is 4.782592536 . The rate is calculated by the following formula:

$$
\text { rate }=-\frac{\log (\text { Error }(\text { step } 1) / \text { Error }(\text { step } 2))}{\log (\text { step } 1 / \text { step } 2)} .
$$

Suppose that the rate of convergence of binomial method is $q$; that is,

$$
\begin{aligned}
& \text { Error }(\text { step } 1)=\alpha\left(\frac{T}{\text { step } 1}\right)^{q} \\
& \text { Error }(\text { step } 2)=\alpha\left(\frac{T}{\text { step } 2}\right)^{q} .
\end{aligned}
$$

Then

$$
\begin{gathered}
\log \mid \operatorname{Error}(\text { step } 1) \mid \approx \log (\alpha)+q \log \left(\frac{T}{\text { step } 1}\right) \\
\log |\operatorname{Error}(\operatorname{step} 2)| \approx \log (\alpha)+q \log \left(\frac{T}{\text { step } 1}\right) .
\end{gathered}
$$

So

$$
q=-\frac{\log (\mid \text { Error }(\text { step } 1) / \text { Error }(\text { step } 2) \mid)}{\log (\text { step } 1 / \text { step } 2)}
$$

Example 2. Use capped option as a tool to improve CRR model with parameters: $S 0=100 ; \delta=0.03 ; r=0.03$; $\sigma=0.2 ; T=0.5 ; t=0 ; K=100$. The benchmark value for the American option calculated by CRR binomial tree with 50,000 steps is 5.568079 . 
TABLE 1: Numerical results for Example 1.

\begin{tabular}{lcccccc}
\hline Steps & CRR binomial tree & Error & Rate & Improved binomial tree & Error & Rate \\
\hline 100 & 4.775603311 & -0.00699 & - & 4.787452236 & 0.0048597 & - \\
200 & 4.779176352 & -0.00342 & 1.032747 & 4.785155998 & 0.002563463 & 0.922773287 \\
400 & 4.780890888 & -0.0017 & 1.005453 & 4.78396397 & 0.001371434 & 0.902408294 \\
800 & 4.781760011 & -0.00083 & 1.031369 & 4.783305899 & 0.000713364 & 0.942975933 \\
1600 & 4.782184168 & -0.00041 & 1.027623 & 4.782971819 & 0.000379283 & 0.911362084 \\
\hline
\end{tabular}

TABLE 2: Numerical results for Example 2.

\begin{tabular}{|c|c|c|c|c|c|c|}
\hline Steps & CRR binomial tree & Error & Rate & Improved binomial tree & Error & Rate \\
\hline 100 & 5.555259412 & -0.012819 & - & 5.57314351 & 0.00506440 & - \\
\hline 200 & 5.561670746 & -0.006408 & 1.0003342 & 5.57065882 & 0.00257971 & 0.97317924 \\
\hline 400 & 5.564883789 & -0.003195 & 1.0039956 & 5.56939269 & 0.00131358 & 0.97370770 \\
\hline 800 & 5.566492564 & -0.001586 & 1.0100720 & 5.56875293 & 0.00067382 & 0.96305278 \\
\hline 1600 & 5.567298182 & -0.000780 & 1.0226286 & 5.56843027 & 0.00035116 & 0.94021553 \\
\hline
\end{tabular}

TABLE 3: Numerical results for Example 3.

\begin{tabular}{lcccccc}
\hline Steps & CRR binomial tree & Error & Rate & Improved binomial tree & Error & Rate \\
\hline 100 & 6.07615754 & -0.013941 & - & 6.09471576 & 0.00461651 & - \\
200 & 6.08313793 & -0.006961 & 1.0019746 & 6.09242930 & 0.00233005 & 0.98644161 \\
400 & 6.08663147 & -0.003467 & 1.0053502 & 6.09128023 & 0.00118098 & 0.98037573 \\
800 & 6.08837907 & -0.001720 & 1.0114551 & 6.09070422 & 0.00060497 & 0.96504590 \\
1600 & 6.08925308 & -0.000846 & 1.0235380 & 6.09041584 & 0.00031659 & 0.93422088 \\
\hline
\end{tabular}

Example 3. Use capped option as a tool to improve CRR model with parameters: $S 0=100 ; \delta=0.03 ; r=0.07$; $\sigma=0.2 ; T=0.5 ; t=0 ; K=100$. The benchmark value for the American option calculated by CRR binomial tree with 50,000 steps is 6.090099249 .

From Tables 1, 2, and 3, it is clear that all the errors calculated by improved binomial tree method are smaller than the errors of the CRR binomial tree method. According to Examples 1 and 3, the value of American option calculated by improved binomial tree method with 100 steps is better than that calculated by traditional binomial tree method with 200 steps. And capped option does a better job in improving the CRR binomial tree method for the higher dividend and lower dividend CRR model than the CRR model with dividend being equal to risk free rate according to Tables 1-3.

The following example tests the capped option as a tool to improve the traditional binomial tree method in Tian model [8]. The numerical result shows that this improvement can be also used in Tian model.

Example 4. Use capped option as a tool to improve Tian model with parameters: $S 0=100 ; \delta=0.07 ; r=0.03$; $\sigma=0.2 ; T=0.5 ; t=0 ; K=100$. The benchmark value for the American option calculated by Tian binomial tree with 50,000 steps is 4.78259298824482 .

\subsection{New LSM}

Example 5. Use capped option as a tool to improve LSM. Consider the parameters:

$$
\begin{gathered}
r=0.03, \quad \sigma=0.2, \quad \delta=0.07, \\
K=100, \quad N=20, \quad P=50,000, \quad T=0.5 .
\end{gathered}
$$

Let $a=0,0.25,0.5,0.75, b=1.125$; take interval $[a *$ $\left.L^{*}, b * L^{*}\right]$ to reduce the paths. The initial values of underlying asset: $S 0=\left[\begin{array}{lllll}80 & 90 & 100 & 110 & 120\end{array}\right]$. The benchmark values, which are calculated using 500,000 paths, are: $\bar{V}=$ [0.219 1.3864 .78311 .09820 .000$]$. The output parameters showed by Tables $5,6,7,8$, and 9 are

$V(i, j)$ : the value of options, where $i=1, \ldots, 5$ and $j=1, \ldots, 5$;

$\operatorname{Res}(i, j):$ the error, where Res $=\sqrt{\sum_{k=1}^{n}\left(V_{k}-\bar{V}\right)^{2}} / n$, and $n=100$;

Reduced path $(i, j)$ : the number of average reduced paths;

$\operatorname{Var}(i, j):$ the average variance;

$\operatorname{Avt}(i, j):$ the average time.

We simulate 100 times to get the output parameters. 
TABle 4: Numerical results for Example 4.

\begin{tabular}{lcccccc}
\hline Steps & Tian binomial tree & Error & Rate & Improved binomial tree & Error & Rate \\
\hline 100 & 4.775829512 & 0.0067634 & - & 4.787677204 & 0.00508421 & - \\
200 & 4.779289543 & 0.0033034 & 1.0337934 & 4.785271837 & 0.00267884 & 0.92441183 \\
300 & 4.780385169 & 0.0022078 & 0.9938233 & 4.784452498 & 0.00185950 & 0.90038471 \\
400 & 4.780947507 & 0.0016454 & 1.0218663 & 4.784022516 & 0.00142952 & 0.9140937 \\
500 & 4.781290534 & 0.0013024 & 1.0476757 & 4.783750356 & 0.00115736 & 0.94645693 \\
600 & 4.78151052 & 0.0010824 & 1.0147265 & 4.783567205 & 0.00097421 & 0.94486630 \\
700 & 4.781671345 & 0.0009216 & 1.0433990 & 4.783434838 & 0.00084185 & 0.94733429 \\
800 & 4.781788327 & 0.0008046 & 1.0165205 & 4.783334956 & 0.00074196 \\
900 & 4.781878368 & 0.0007146 & 1.0075284 & 4.783260037 & 0.00066704 & 0.90371784 \\
1000 & 4.781950499 & 0.0006424 & 1.0098803 & 4.783199437 & 0.00060644 & 0.90397488 \\
1100 & 4.782010723 & 0.0005822 & 1.0326610 & 4.783148553 & 0.00055556 & 0.91947996 \\
1200 & 4.782060177 & 0.0005328 & 1.0200962 & 4.783105294 & 0.00051230 \\
1300 & 4.782102211 & 0.0004907 & 1.0266497 & 4.783068897 & 0.93163122 \\
1400 & 4.782138982 & 0.0004540 & 1.0509063 & 4.783037294 & 0.00047590 & 0.92069405 \\
1500 & 4.782170649 & 0.0004223 & 1.0479512 & 4.783010233 & 0.00044430 & 0.92722175 \\
1600 & 4.782198327 & 0.0003946 & 1.0502588 & 4.78298612 & 0.00041724 & 0.91082254 \\
\hline
\end{tabular}

TABLE 5: Numerical result for Example 5.

\begin{tabular}{lccccc}
\hline$V(i, j)$ & $S 0=80$ & 90 & 100 & 110 & 120 \\
Standard LSM & 0.2027 & 1.3485 & 4.6388 & 11.0304 & 19.9684 \\
{$[a, b]=[0,1.125]$} & 0.2030 & 1.3551 & 4.6219 & 11.0805 & 19.9684 \\
{$[a, b]=[0.25,1.125]$} & 0.1795 & 1.3843 & 4.4811 & 10.7401 & 19.0541 \\
{$[a, b]=[0.5,1.125]$} & 0.1795 & 1.3843 & 4.4683 & 10.6162 & 18.2787 \\
{$[a, b]=[0.75,1.125]$} & 0.1795 & 1.3843 & 4.4683 & 10.6162 & 18.2787 \\
\hline
\end{tabular}

TABLE 6: Numerical result for Example 5.

\begin{tabular}{lrcccc}
\hline $\operatorname{Res}(i, j)$ & $S 0=80$ & 90 & 100 & 110 & 120 \\
Standard LSM & 0.0010 & 0.0029 & 0.0094 & 0.0263 & 0.0027 \\
{$[a, b]=[0,1.125]$} & 0.0010 & 0.0028 & 0.0089 & 0.0261 & 0.0028 \\
{$[a, b]=[0.25,1.125]$} & 0.0010 & 0.0031 & 0.0101 & 0.0269 & 0.0508 \\
{$[a, b]=[0.5,1.125]$} & 0.0010 & 0.0032 & 0.0105 & 0.0313 & 0.0772 \\
{$[a, b]=[0.75,1.125]$} & 0.0010 & 0.0032 & 0.0105 & 0.0313 & 0.0772 \\
\hline
\end{tabular}

TABLE 7: Numerical result for Example 5.

\begin{tabular}{lccccc}
\hline Reduced path $(i, j)$ & $S 0=80$ & 90 & 100 & 110 & 120 \\
Standard LSM & 0 & 0 & 0 & 0 & 0 \\
{$[a, b]=[0,1.125]$} & 0.1 & 4.4 & 30.7 & 148.4 & 442.1 \\
{$[a, b]=[0.25,1.125]$} & 27.4 & 237.5 & 1078.7 & 1995.6 & 2267.9 \\
{$[a, b]=[0.5,1.125]$} & 27.4 & 237.5 & 1079 & 2002.8 & 2318.8 \\
{$[a, b]=[0.75,1.125]$} & 27.4 & 237.5 & 1079 & 2002.8 & 2318.8 \\
\hline
\end{tabular}

Table 5 shows that improved LSM is right. Tables 6 and 8 show that when $[a, b]=[0,1.125]$, the Res and the average variance are both improved. Table 7 shows that the reduced paths increase when the initial asset price goes larger. We can see from Table 9 that the times of calculation are much reduced. According to Tables 5, 6, 7, 8, and 9, improved LSM is faster and meanwhile does not decrease the accuracy.
TABle 8: Numerical result for Example 5.

\begin{tabular}{lccccc}
\hline $\operatorname{Var}(i, j)$ & $S 0=80$ & 90 & 100 & 110 & 120 \\
Standard LSM & 0.0120 & 0.0120 & 0.0563 & 0.0565 & 0.0265 \\
{$[a, b]=[0,1.125]$} & 0.0119 & 0.0353 & 0.0551 & 0.0573 & 0.0265 \\
{$[a, b]=[0.25,1.125]$} & 0.0123 & 0.0428 & 0.0793 & 0.1183 & 0.1341 \\
{$[a, b]=[0.5,1.125]$} & 0.0123 & 0.0428 & 0.0791 & 0.1209 & 0.1488 \\
{$[a, b]=[0.75,1.125]$} & 0.0123 & 0.0428 & 0.0791 & 0.1209 & 0.1488 \\
\hline
\end{tabular}

TABLE 9: Numerical result for Example 5.

\begin{tabular}{lccccc}
\hline $\operatorname{Avt}(i, j)$ & $S 0=80$ & 90 & 100 & 110 & 120 \\
Standard LSM & 0.0011 & 0.0008 & 0.0017 & 0.0045 & 0.0072 \\
{$[a, b]=[0,1.125]$} & 0.0003 & 0.0006 & 0.0019 & 0.0045 & 0.0064 \\
{$[a, b]=[0.25,1.125]$} & 0.0005 & 0.0006 & 0.0006 & 0.0008 & 0.0013 \\
{$[a, b]=[0.5,1.125]$} & 0.0003 & 0.0002 & 0.0003 & 0.0006 & 0.0005 \\
{$[a, b]=[0.75,1.125]$} & 0.0003 & 0.0002 & 0.0006 & 0.0003 & 0.0003 \\
\hline
\end{tabular}

Especially when we use $[a, b]=[0,1.125]$ to reduce the paths, the result is much better and faster.

\section{Conclusions}

We used the capped option as a tool to improve the binomial tree method and LSM method. From the above examples, we conclude that capped option does a good job in improving both the binomial tree and LSM. According to Tables 1-3, the improved binomial tree method performs well for CRR models. Table 4 shows that it works well for Tian model. It is more efficient to replace the values of American call option one step before maturity with the values of corresponding American capped call options. Tables 5-9 give the numerical result of the improved LSM. By filtering out the invalid paths with the lower bound, the LSM method has been improved. According to above result, the capped option is a very useful 
tool in improving the traditional binomial tree method and LSM. This paper only studied two basic methods. Other more complex models, such as stochastic models and multiple assets models, need to be studied in the future.

\section{Conflict of Interests}

The authors declare that there is no conflict of interests regarding the publication of this paper.

\section{Acknowledgments}

The work was supported by Program for New Century Excellent Talents in University (no. NCET-12-0922). The authors thank Mr. Zhidong Wang for the implementation of Section 5.2.

\section{References}

[1] J. C. Cox, S. A. Ross, and M. Rubinstein, "Option pricing: a simplified approach," Journal of Financial Economics, vol. 7, no. 3, pp. 229-263, 1979.

[2] Y. K. Kwok, Mathematical Models of Financial Derivatives, Springer, Singapore, 1998.

[3] F. A. Longstaff and E. S. Schwartz, "Valuing American options by simulation: a simple least-squares approach," Review of Financial Studies, vol. 14, no. 1, pp. 113-147, 2001.

[4] P. P. Boyle and S. M. Turnbull, "Pricing and Hedging Capped Options," Journal of Futures Markets, vol. 9, pp. 41-54, 1989.

[5] M. Broadie and J. Detemple, "American capped call options on dividend-paying assets," Review of Financial Studies, vol. 8, pp. 161-191, 1995.

[6] M. Broadie and J. Detemple, "American option valuation: new bounds, approximations, and a comparison of existing methods," Review of Financial Studies, vol. 9, no. 4, pp. 1211-1250, 1996.

[7] J. M. Harrison and S. R. Pliska, "Martingales and stochastic integrals in the theory of continuous trading," Stochastic Processes and their Applications, vol. 11, no. 3, pp. 215-260, 1981.

[8] Y. Tian, "Modified lattice approach to option pricing," Journal of Futures Markets, vol. 13, pp. 563-577, 1993. 


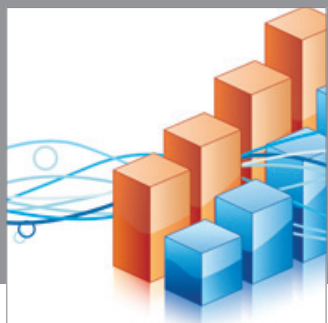

Advances in

Operations Research

mansans

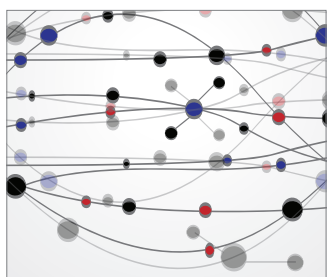

The Scientific World Journal
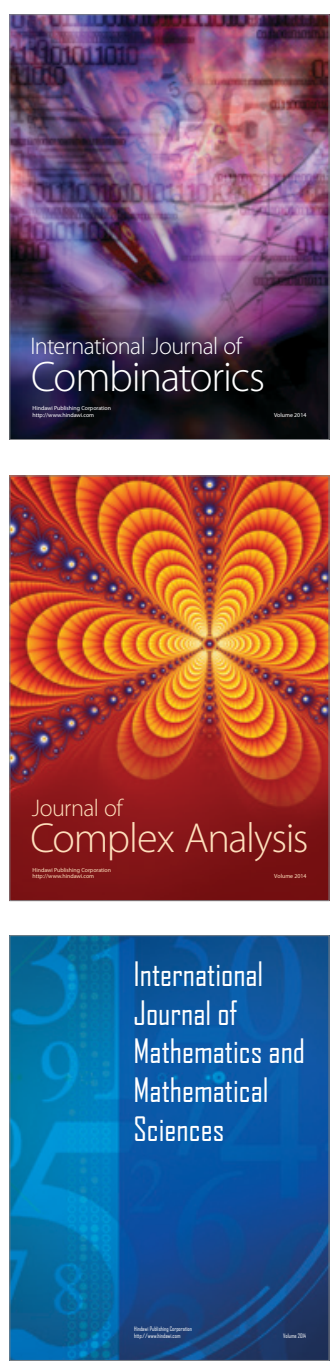
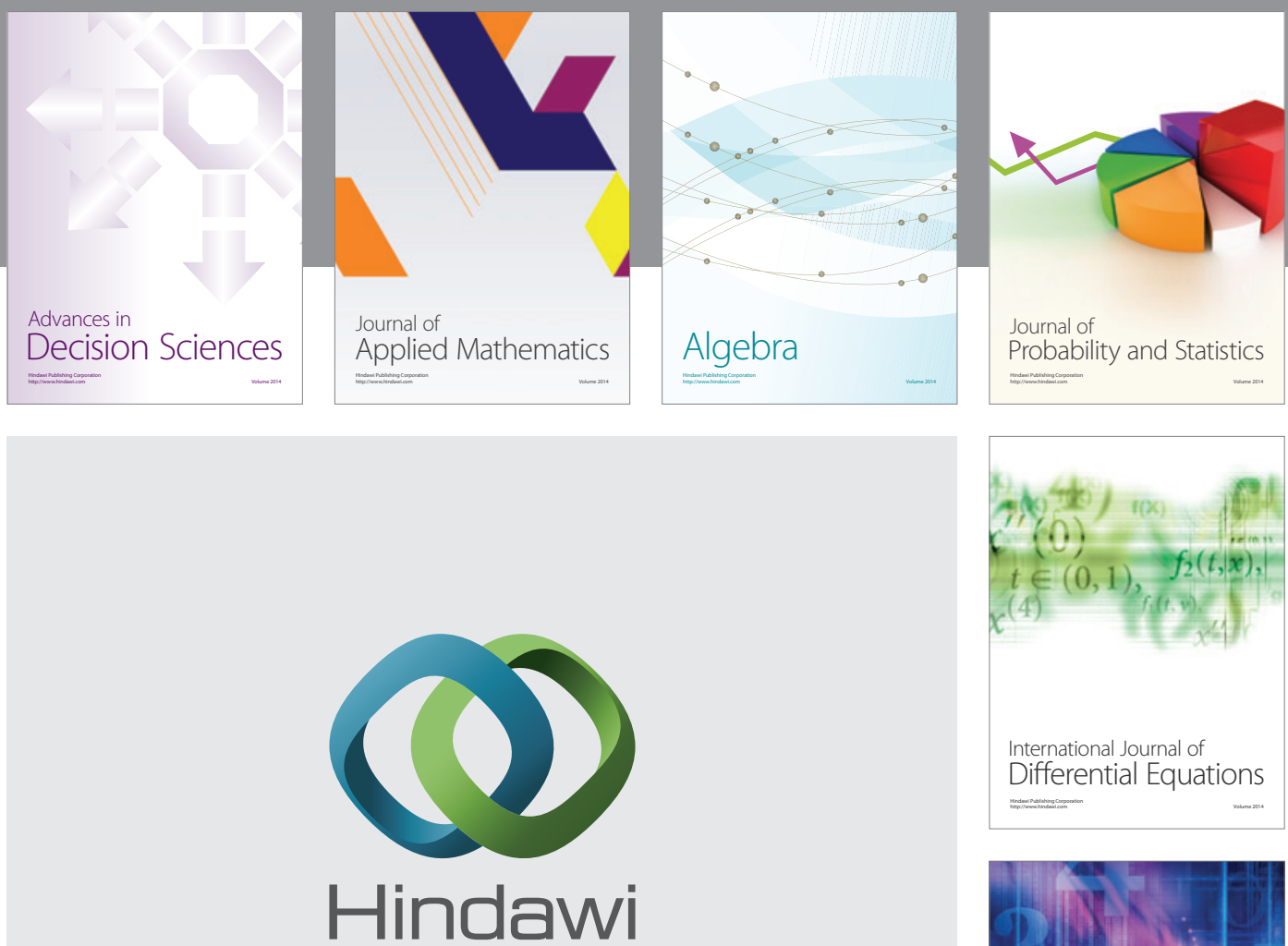

Submit your manuscripts at http://www.hindawi.com
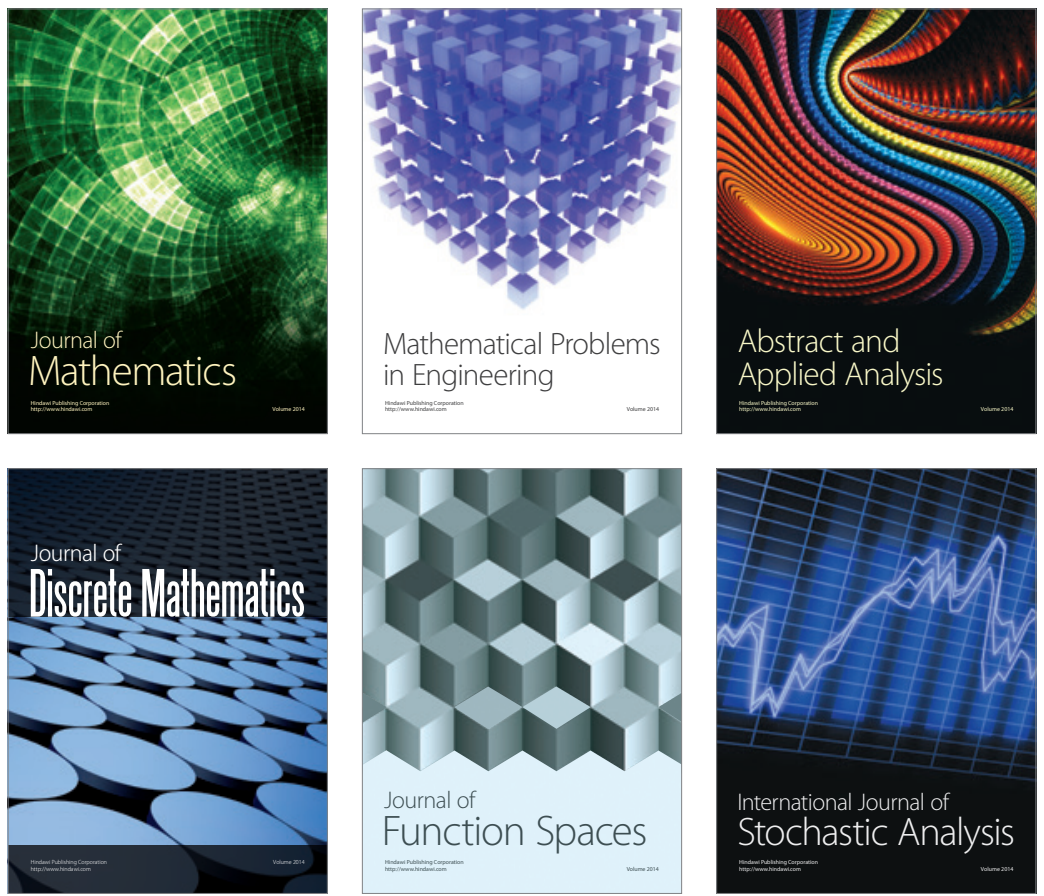

Journal of

Function Spaces

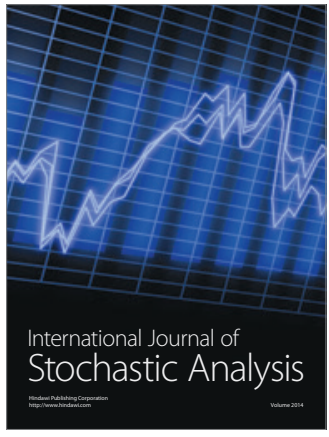

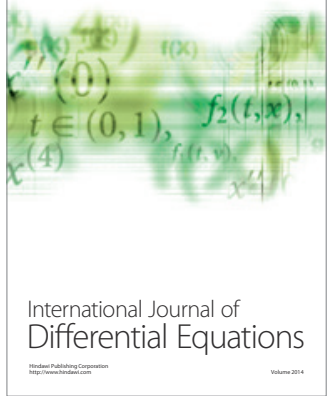
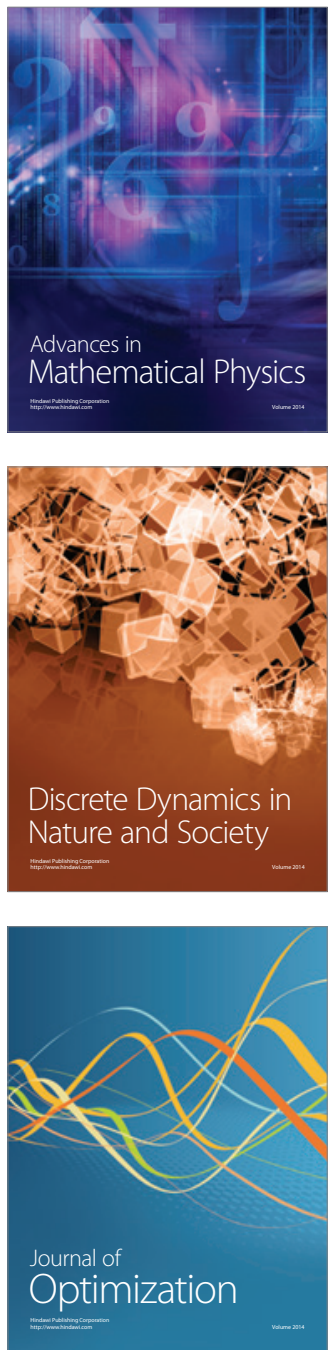